\title{
Real-world evidence: A review of clinical experience in metastatic gastric cancer second-line treatment with ramucirumab
}

\author{
Miguel Quintana-Quintana ${ }^{1, *}$, María Consuelo Díaz-Romero², \\ Christian Patricio Camacho-Limas ${ }^{3}$, Laura Torrecillas-Torres ${ }^{4}$, Erika Ruiz-García², \\ José Antonio Acevedo-Delgado ${ }^{5}$, Guillermo Olivares-Beltrán ${ }^{3}$, Francisco Medina-Soto ${ }^{6}$, \\ German Calderillo-Ruiz ${ }^{2}$, Jorge Alberto Robles-Aviña ${ }^{7}$, Pedro Sobrevilla ${ }^{8}$, Juan Cruz-Baca ${ }^{9}$, \\ Alicia Espinoza-Acosta ${ }^{10}$, Daniel Motola-Kuba ${ }^{11}$, Ramiro Magaña-Serrano ${ }^{12}$, \\ Juan Paulo Ceja-García ${ }^{13}$, Alejandro Molina-Franco ${ }^{14}$, Leticia Vázquez-Cortes ${ }^{15}$, \\ Angelina Sáenz-Frías ${ }^{16}$, Iván Romerico González-Espinoza ${ }^{17}$, Eduardo Téllez-Bernal ${ }^{18}$, \\ Eva Lucia Willars-Inman ${ }^{19}$, Luis Javier Barajas-Figueroa ${ }^{20}$, Flor de The Bustamante-Valles $^{21}$, \\ Benjamín Dávalos-Félix ${ }^{22}$, Saúl Campos-Gómez ${ }^{23}$
}

\begin{abstract}
${ }^{1}$ Department of Medical Oncology, Hospital General Naval de Alta Especialidad, Coyoacán, Ciudad de México; ${ }^{2}$ Instituto Nacional de Cancerología (INCAN), Ciudad de México; ${ }^{3}$ Centro Médico ABC, Campus Observatorio, Ciudad de México; ${ }^{4}$ Centro Médico Nacional 20 de Noviembre, Ciudad de México; ${ }^{5}$ Medicina Privada, Guadalajara, Jal.; ${ }^{6}$ Centro de Especialidades Médicas, Monterrey, N.L.; ${ }^{7} \mathrm{Hospital}$ Central Sur de Alta Especialidad PEMEX, Ciudad de México; ${ }^{8} \mathrm{Hospital}$ Ángeles del Pedregal, Ciudad de México; ${ }^{9} \mathrm{Hospital}$ Christus Muguerza, Ciudad de México; ${ }^{10} \mathrm{Hospital}$ General 5 de Diciembre, ISSSTE, Mexicali, B.C.; ${ }^{11} \mathrm{Hospital}$ Médica Sur, Ciudad de México; ${ }^{12}$ Centro Médico de Especialidades, Cd. Juárez, Chih.; ${ }^{13}$ Hospital General, La Paz, B.C.S.; ${ }^{14}$ Oncología Médica, Mérida, Yuc.; ${ }^{15} \mathrm{Hospital}$ General de México, Ciudad de México; ${ }^{16}$ UMAE IMSS 25, N.L.; ${ }^{17} \mathrm{Hospital}$ Ángeles, Puebla, Pue.; ${ }^{18} \mathrm{Hospital}$ ISSSTEP, Puebla, Pue.; ${ }^{19}$ ISSSTE, Saltillo, Coah.; ${ }^{20} \mathrm{Hospital}$ Star Médica Ciudad Juárez, Cd. Juárez, Chih.; ${ }^{21} \mathrm{COC}$ Centro Oncológico de Chihuahua, Cd. Juárez, Chih.; ${ }^{22}$ Práctica Privada, Monterrey, N.L.; ${ }^{23}$ ISSEMYM Centro Oncológico Estatal, Toluca de Lerdo, Edo. de México. México.
\end{abstract}

Received for publication: 6 August 2016; accepted for publication: 25 February 2017 Available online: 6 November 2017

\section{KEY WORDS \\ Gastric cancer; \\ Stomach cancer; \\ Metastatic gastric \\ cancer; \\ Advanced gastric \\ cancer; \\ Ramucirumab; \\ Second-line; \\ Antiangiogenic; \\ Real-world evidence}

\begin{abstract}
An improvement in overall survival of patients with gastrointestinal tumors has been observed in recent years, with main factors for this being an in-depth understanding of molecular biology, determination of prognostic factors, and of course, the development of new drugs, all of which has allowed gradual but significant advances in the treatment of gastric cancer. First-line treatment has shown benefits; however, many of these patients show disease progression, which raises the challenge of optimizing the ensuing treatment lines and developing better treatment strategies. The role of second-line treatment has recently been introduced and discussed, although there is evidence regarding the benefit of some drugs such as docetaxel, paclitaxel and irinotecan in second-line treatment, with this benefit having shown a reduction of approximately $18 \%$ in the risk of death, there is still a need to discuss and analyze efficacy in overall survival, progression-free survival and toxicity profile, with a special emphasis on the appropriate choice of drugs according to efficacy, toxicity, prognostic factors, and patient characteristics. The objective of this cross-sectional study is
\end{abstract}

*E-mail for correspondence: quintanaqm@hotmail.com (M. Quintana-Quintana) 
to analyze medical experience in real-life clinical practice in the setting of second-line treatment for metastatic gastric cancer in patients treated with ramucirumab, as well as to analyze prognostic factors and their impact on everyday clinical practice. In recent years, an improvement in overall survival has been observed in patients with gastrointestinal tumors; some factors that explain this include deep knowledge of molecular biology, determination of prognostic factors and, of course, and the development of new drugs. All of this has allowed gradual but significant advances in the treatment of gastric cancer. One of the greatest challenges posed by this disease is that, in spite of all therapeutic advances that have been achieved, gastric cancer remains the third cause of cancer mortality worldwide ${ }^{1}$. Since more than $70 \%$ of cases are identified at advanced stages, in spite of first-line treatment having shown benefits, many of these patients experience disease progression; this poses the challenge of optimizing subsequent therapeutic lines and developing improved therapies ${ }^{2,3}$. The role of second-line therapy has recently been introduced and reviewed. Despite the fact that some evidence points to the benefits of some drugs, such as docetaxel, paclitaxel, and irinotecan in second-line treatment, a benefit that has shown a decrease of almost $18 \%$ in the risk of death, efficacy has yet to be examined in terms of overall survival, progression-free survival and toxicity profile, with special emphasis on adequate drug selection based on efficacy, toxicity, prognostic factors, and patient characteristics ${ }^{2,3}$. At present, this need has led to the development of new therapeutic measures to achieve better results and incorporate new molecular targets ${ }^{4}$. The purpose of this cross-sectional study is to examine medical experience in real-life clinical practice, in the setting of second-line treatment of metastatic gastric cancer in patients treated with ramucirumab, and to describe prognostic factors and their impact on real-life clinical practice. (creativecommons.org/licenses/by-nc-nd/4.0/).

\section{INTRODUCTION}

Gastric cancer (GC) is the third cause of cancer-related death worldwide. Around 1 million new cases are diagnosed every year ${ }^{1}$. GLOBOCAN 2012 statistics record GC diagnosis in 930,000 individuals annually. Incidence shows the strong influence of ethnic and geographic factors: It is higher in Asia, East Europe, and South America, while incidence rates reported in Africa and North America are lower ${ }^{2,3}$. GC in México is a public health problem and the third cause of cancer-related deaths from the third decade of life on, with a trend that does not decline over time ${ }^{4,5}$. A GC mortality rate of 6.4 cases per 100,000 population has been reported in men, as compared with 4.7 cases per 100,000 in women 6 .

In spite of advances regarding the knowledge of molecular biology and surgical techniques, improvements in diagnosis and development of new chemotherapy protocols, clinical outcome for patients with GC is generally limited, with a 5 -year survival rate of $5-20 \%$ and an average expectancy of 10-12 months as median overall survival in subjects with metastatic disease; this is largely due to the fact that a high percentage of patients, from $65 \%$ to $90 \%$, is diagnosed at non-resectable and metastatic stages, where the only therapeutic option is chemotherapy ${ }^{7,8}$.

Palliative chemotherapy for advanced disease was a topic of discussion for a long time. A meta-analysis by Wagner concluded that, in terms of efficacy, a significant improvement in overall survival is observed with regimens including fluoropyrimidines, cisplatin, and anthracyclines (hazard ratio [HR], 0.82; 95\% confidence interval [Cl], 0.73-0.92) ${ }^{9}$.

This same meta-analysis showed that doublets are more efficacious than monotherapy alone, and considered doublets based on fluoropyrimidines plus a platinum-based salt as standard therapy.

With regard to the role of triplets in the treatment of advanced or metastatic GC, it should be noted that multiple studies have been conducted with the purpose to examine whether the addition of a third drug may have a positive effect on mean survival, either with anthracyclines or taxanes. An improvement in response rates and disease-free survival has been shown, albeit with higher toxicity, which requires dose adjustments to improve the toxicity profile, and these combinations have, therefore, been proposed as a therapeutic possibility in very selected patients with adequate performance status ${ }^{10,11}$.

However, the toxicity profile, as well as the performance status grade and comorbilities of the patient should always be considered when choosing a therapeutic regimen ${ }^{11}$.

In spite of the advances in first-line treatment, many patients experience progression, and nearly $40 \%$ of these patients have to undergo a second line of rescue treatment ${ }^{12}$.

The first attempts to offer an alternative for these patients were based on Phase II and III studies, where single agent chemotherapy was compared with the best supportive care. It is important taking into account the small patient populations included in these studies, the design and the heterogeneity of the population ${ }^{13-15}$.

An attempt to incorporate the knowledge on molecular pathways to treatment measures has been made in recent years. As a consequence, several signaling pathways have been identified that can be incorporated to optimal care of patients. It should be noted that GC carcinogenesis is complex and has not been fully characterized. Many signaling pathways have been implicated, including the signaling pathways targeting the epidermal growth factor receptor (EGFR), signaling pathways targeting anti-EGFR, tyrosine kinase inhibitors, signaling pathways targeting anti-HER2, signaling pathways targeting CMET pathway overexpression, signaling pathways targeting mammalian target of rapamycin, signaling pathways for checkpoint inhibitors, and vascular endothelial growth factor (VEGF) signaling pathways, which have been examined to incorporate these molecular targets to the treatment of $\mathrm{GC}^{16,17}$.

Comprehensive knowledge of signaling pathways enabled the development of several clinical trials with molecular targets, many of which have failed with regard to their re- 
sults, including the following drugs cetuximab, panitumumab, lapatinib, gefitinib, erlotinib, and everolimus, which unfortunately were unable to show an improvement in terms of overall survival.

The knowledge of GC molecular profile has enabled the examination of more promising signaling pathways, which opens new opportunities for the treatment of GC. However, as previously mentioned, therapeutic progress has been limited, with the exception of two targeted therapies. One of these is trastuzumab as first-line treatment of GC, supported by the ToGA trial, which enrolled patients with GC or non-resectable or metastatic gastro-esophageal junction cancer with HER2 overexpression (22\%), and the results of which regarding survival found benefit favoring this drug. An overall survival of 13.8 months was reported with trastuzumab in comparison with 11.1 months for chemotherapy alone, and progression-free survival (PFS) of 6.7 in comparison with 5.5 months ${ }^{18}$.

The other therapeutic approach that has shown an effect on survival in patients with GC is the inhibition of angiogenesis signaling pathways, where multiple preclinical trials have shown an improvement in tumor growth control and metastases progression in GC through the inhibition of the VEGF pathway ${ }^{19}$.

Several clinical trials were carried out with drugs aiming to inhibit angiogenesis, with the purpose to explore this signaling pathway. However, compounds such as bevacizumab, aflibercept, axitinib, orantinib, pazopanib, regorafenib, telatinib, and vandetanib have not shown positive results. So far, the only angiogenesis-inhibiting drug with proven efficacy and positive effects on survival has been ramucirumab. This drug is a fully humanized monoclonal antibody that targets the VEGF receptor ${ }^{20}$.

Two large clinical trials have evaluated the efficacy of ramucirumab in terms of overall survival in patients progressing to a first-line of treatment: REGARD and RAINBOW. These trials show the most robust data, with more adequate available evidence for second lines, since they included large numbers of patients and are appropriately designed. They are adequately conducted, double-blind, placebo-controlled trials that achieved the primary endpoint of overall survival improvement and also showed a consistent improvement in PFS, while maintaining the quality of life $\mathrm{e}^{21,22}$.

The REGARD trial assessed ramucirumab as second-line treatment in patients with advanced GC or cancer of the gastro-esophageal junction; mean survival was 5.2 months for the antibody treatment and 3.8 months in the control group ( $P=0.0047)$. Greater PFS was also reported for ramucirumab (2.1 months, compared with 1.3 months with placebo). These results revealed that ramucirumab is the first biologic treatment with a proven benefit on survival after progression with a first-line of therapy ${ }^{21}$.

A second trial (RAINBOW) investigated the same antibody in combination with weekly paclitaxel, compared to a taxane and best supportive medical care as second-line therapy in patients with gastric and gastro-esophageal cancers that had progressed on a first-line of treatment with chemotherapy. Survival was significantly longer in the ramucirumab plus paclitaxel group in comparison with the control group (9.6 vs. 7.4 months).

Ramucirumab plus paclitaxel delayed disease progression (PFS of 4.4 vs. 2.9 months) and showed a higher response rate ( 28 vs. $16 \%)^{22}$. Based on these results, regulatory authorities approved the drug as monotherapy or in combination with paclitaxel in patients with advanced or metastatic gastro-esophageal junction or GC after progression, over fluoropyrimidine or platinum-based schemes.

Ramucirumab is the first compound approved for the treatment of advanced or metastatic GC after first-line therapeutic failure. If the abovementioned information is considered, it is important to have local experience available regarding population characteristics, as well as treatment results, and prognostic factor identification, which allows for the therapeutic approach and its results in GC to be improved.

Therefore, as part of SMeO initiatives, an epidemiological, observational, cross-sectional study was conducted by means of a self-administered questionnaire, designed for the purpose of this study, and administered as a survey during the period of January 12 to 27, 2017. Primary interest events included population epidemiological characteristics, number of received treatment cycles, total treatment duration (months), and PFS (months). Frequency of Grade 3 and 4 toxicity and overall treatment response (partial response, stable disease, or complete response) were also evaluated.

\section{RESULTS}

A total of 66 patients were included, out of which a total of 58 were analyzed, since eight patients were excluded because they had initiated therapy not even completing two cycles of the scheme.

When demographic data were analyzed, mean age for the 58 patients was found to be 56 years; in this respect, age showed the following distribution: $32.8 \%$ were $28-48$ years old and $34.4 \%$ were $49-63$ years old.

Regarding distribution by age, median age at disease onset was found to be lower than that reported in the medical literature, with a mean of 64 years. Conversely, $32.8 \%$ of patients were 48 years old (Fig. 1). Gender distribution was as follows: females, $37.9 \%$; males, $62.1 \%$.

With regard to the ECOG score reported at the beginning of second-line therapy, the following distribution was identified: ECOG 0, 14\%; ECOG 1, 65\%; ECOG 2, 21\%; ECOG 3 patients were not included.

Undoubtedly, one of the most relevant prognostic factors is the ECOG performance status (PS) scores, a parameter that is widely used as a predictor related to treatment. An ECOG of 0 to 1 has been previously shown to be considered the best predictor when compared with patients with an ECOG of 2-3 (poor performance status). One of the trials showing the effect of the ECOG score on mean survival is that of Lee and colleagues, which examined 1,455 patients with GC on first-line therapy; this study concluded that in patients with an ECOG > 2 the survival rate was $17.1 \%$, in comparison with $39.2 \%$ in patients with an ECOG of 0 to $1^{23}$.

In this study, the authors found that performance status (ECOG) was correlated with PFS and that patients with an ECOG of 0 exhibited a PFS period of 5 months, in contrast with those with an ECOG of 2, where PFS was 3 months. This revealed, once again and without a doubt that ECOG remains a highly relevant prognostic factor.

In the report by Catalano et al., which specifically examined the influence of pathological clinical factors on survival in patients on second-line chemotherapy for GC, a 1-year 


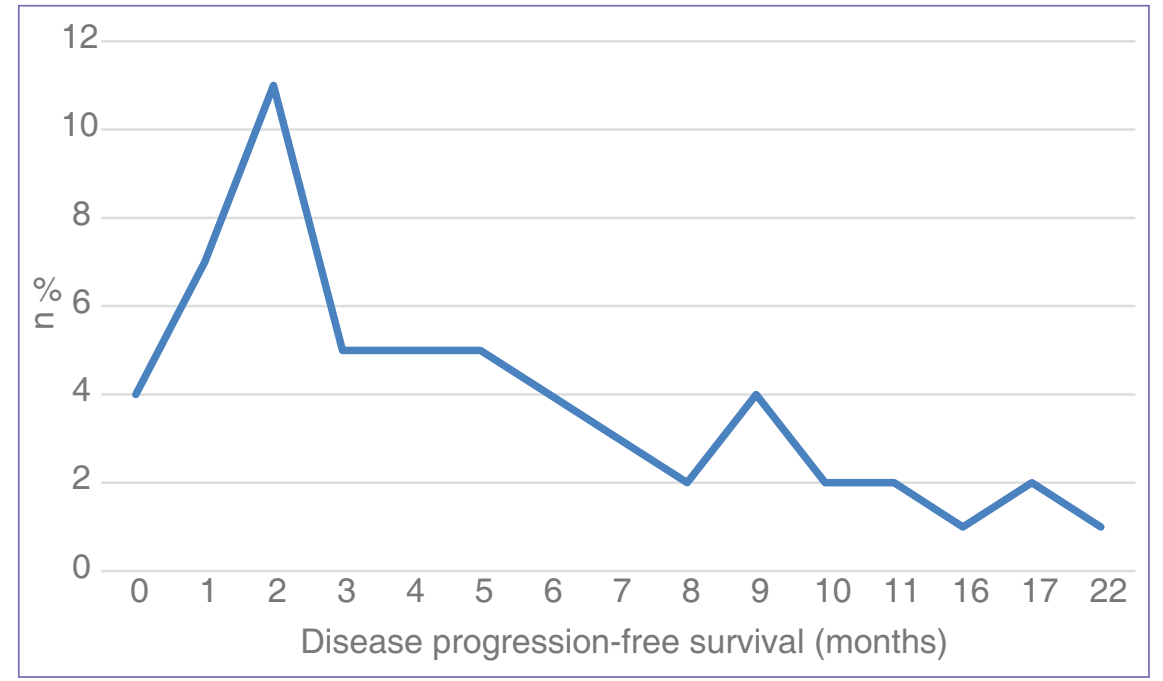

Figure 1. Disease progression-free survival (months) in the study sample $(n=61), 2017$.

survival of $25 \%$ was reported in patients with an ECOG of 0 to 1 , in comparison with $8 \%$ in subjects with an ECOG of 2 , who had two- to three-fold more probabilities of dying within one year (HR, 1.79 [1.16-2.77]; $P=0.008)^{24}$.

As for histological types, the intestinal type prevailed in 31 patients $(54 \%)$, followed by diffuse and mixed types in 18 and 9 patients (30\% and 16\%), respectively. The proportions of patients with poorly and moderately differentiated tumors were similar: $48 \%$ of cases were Grade 1 and 2 adenocarcinomas; only 3 patients (4\%) had highly differentiated tumors. No link was identified between histological type and degree of differentiation and PFS.

Previous trials have identified metastatic sites as prognostic factors. For example, the study by Wang and colleagues showed that the presence of liver and bone metastases was an independent prognostic factor, even in patients with adequate performance status. Both these metastatic sites are poor prognostic factors. The authors of this retrospective series of 310 patients assigned to first-line treatment with chemotherapy found that patients with metastases in more than two organs experienced a worse effect on survival in comparison with those with metastasis to a single organ (HR, 1.47; 95\% Cl, 1.11-1.96; $P=0.007)^{25}$.

In the study by the above-mentioned authors, metastatic disease (Stage IV) was observed in all patients receiving first-line treatment with chemotherapy, and 30 of them (49\%) had multiple metastatic sites: 0 to 1 site was found in $27.6 \%$ of cases; 1 to 2 sites in $17.2 \%$; and 2 to 3 metastatic sites in $55.2 \%$.

The most common metastatic site was the liver (46\%), followed by the peritoneum, retroperitoneal lymph nodes and the lung. A trend toward a longer PFS period was identified in patients with 0 to 1 metastasis, although this finding was not conclusive owing to the sample size.

The same study reported the presence of carcinomatosis in $60.3 \%$, although no relationship as a prognostic factor was found. With regard to ascites, it was observed in $36.2 \%$ of patients.

Weight loss prior or during chemotherapy has been noted in several cancers, including GC, and it is generally associated with an attenuated tolerance to treatment, which affects the strength of the chemotherapeutic dosage. In the study by Ock et al. that examined 719 patients, mean survival in subjects receiving palliative chemotherapy as first-line treatment with a weight loss of $3 \%$ during the $1^{\text {st }}$ month of therapy was significantly shorter in comparison with patients with no weight loss $(9.7 \text { vs. } 16.3 \text { months; } P=0.001)^{26}$.

Notwithstanding, weight loss is not mentioned as a factor in prognostic scales. In the above-mentioned study, the authors observed that a weight loss $>10 \%$ in $50 \%$ of the sample showed a correlation between PFS and absence of weight loss, with an improvement in PFS to 6.9 months in comparison with the subgroup with $>10 \%$ weight loss, which reported 3.8 months. However, these results are not statistically significant owing to the patient population size.

\section{Number of treatment cycles}

With regard to treatment duration, overall median treatment cycles with ramucirumab per patient were $4.0(95 \% \mathrm{Cl}, 3.1-5.0)$.

\section{TREATMENT RESPONSE AND QUALITY OF LIFE}

\section{PFS}

PFS (Fig. 1) in the entire study sample was 4.0 months $(95 \%$ $\mathrm{Cl}, 2.5-5.5)$. In the stratified analysis by age, the largest median was observed among participants aged from 70 to 89 years $(6.0$ months; $95 \% \mathrm{Cl}, 1.0-12.0)$. However, differences between age groups were not statistically significant.

Regarding of toxicities none Grade 3 or 4 was reported, the most common toxicity reported was hypertension.

\section{DISCUSSION}

GC is a highly common neoplasm in underdeveloped countries, with practically comparable incidence and lethality. In Mexico, it constitutes an important cause of death and occupies the first place in gastrointestinal tumors. This lethal prognosis is due to late diagnosis and advanced sta- 
ges at diagnosis in more than $80 \%$ of cases; consequently, therapeutic options are merely palliative in patients suitable for systemic oncologic management. At progression after receiving a first-line of chemotherapy, a portion of these patients (nearly one third) is eligible for second-line treatment. However, this is a difficult challenge in clinical practice because patients are generally fragile, almost all of them compromised in terms of nutritional status. In addition, evidence of a benefit with second-line treatment schemes is relatively recent and, furthermore, this is a heterogeneous disease and knowledge on its biological and molecular behavior has emerged only recently in investigations to identify different GC groups. These treatment approaches have the purpose to improve prognosis and, in the mid-term, to have targeted therapeutic schemes available. These differences may explain treatment response variable effects.

In spite of advances in the knowledge about the biology of GC and the availability of new therapeutic regimens that have led to increases in the parameters of PFS and mean survival in the setting of first-line therapy, the prognosis for patients with GC is still very poor. Second-line treatment is systematically being introduced in many patients, and there are several variants in current clinical practice. This study attempted to evaluate patients exposed to a second-line of treatment with ramucirumab and chemotherapy.

The benefits exhibited by the RFGEV-2 inhibitor, ramucirumab, and the VEGFR-2 tyrosine kinase inhibitor, apatinib, represent an advance in the development of antiangiogenic drugs for metastatic $\mathrm{GC}^{27,28}$. Future trials should investigate ramucirumab given as first-line therapy in combination with other chemotherapeutic agents, or with a specific molecular determination. Therefore, its use as maintenance therapy and the sequence with other active therapies are pending resolution. The choice of chemotherapy, in particular, seems important and demands further investigation to assess combinations with drugs other than taxanes ${ }^{29}$. Thus, performing this type of studies is desirable. Dose strength constitutes an additional problem that should be examined, as should the relationship with exposure, pharmacokinetic concentrations and effectiveness of ramucirumab ${ }^{30}$. On the other hand, it should be pointed out that, in this analysis, most patients had normal weight, which may have related to adequate effectiveness and better tolerance as well as therapeutic compliance.

If increasing costs in oncologic care are considered, a cost-effectiveness analysis is mandatory. In addition, apart from having biomarkers to target these molecular therapies, better clinical tools should be used to select those patients most suitable for these treatments, since we should take into account that the performance status level is a crucial parameter when choosing a therapy. A large proportion of patients herein examined had an ECOG PS of 1 to 2, as in other previously published works. As in the RAINBOW trial, the intestinal type prevailed in this population, although a limiting factor should be mentioned: The number of patients previously exposed to anti-HER therapy was not determined, and the documented proportion of carcinomatosis was lower.

By the same token, tumor burden with regard to metastatic sites has been determined to be a response parameter to second-line therapy.

\section{CONCLUSION}

The treatment of GC continues to pose a challenge due to the heterogeneity of the disease; hence, it is of utmost importance to have reports on real-world clinical experience.

Such reports will allow for a more orderly and systematized identification of population characteristics and prognostic factors in patients treated in regular clinical practice. Therefore, this information makes it possible to have access to more elements contributing to identify the group of patients who will benefit most from a determined therapeutic approach, which will undoubtedly contribute to therapeutic behaviors optimization.

This review concludes that ramucirumab constitutes an adequate second-line treatment in patients with GC to improve time to progression and quality of life.

Although there is no available biomarker for selecting those patients who are eligible for ramucirumab, there are indeed certain clinical parameters such as an ECOG PS of 0-1 and PFS > 6 months, which may constitute appropriate parameters for selecting patients who will be adequate responders and will obtain clinical benefits.

\section{ACKNOWLEDGMENT}

We especially acknowledge the collaboration and information provided for this article by the oncologists who contributed with information from their patients.

\section{REFERENCES}

1. Siegel RL, Miller KD, Jemal A. Cancer statistics, 2016. CA Cancer J Clin. 2016;66:7-30.

2. Ferlay J, Soerjomataram I, Dikshit R, et al. Cancer incidence and mortality worldwide: Sources, methods and major patterns in GLOBOCAN 2012. Int J Cancer. 2015;136:E359-86.

3. Nagano S. Carcinoma of the stomach: A review of epidemiology, pathogenesis, molecular genetics and chemoprevention. World J Gastrointest Oncol. 2012;4:156-69.

4. Sampieri CL, Mora M. Gastric cancer research in Mexico: A public health priority. World J Gastroenterol. 2014;20:4491-502.

5. López-Basave HN, Morales-Vásquez F, Ruiz-Molina JM, et al. Gastric cancer in young people under 30 years of age: Worse prognosis, or delay in diagnosis? Cancer Manag Res. 2013;5:31-6.

6. Registro Histopatológico de Neoplasias Malignas en México. Secretaría de Salud (México), Dirección General de Epidemiología (DGEPI); 2001. Available from: http://www.dgepi.salud.gob.mx/diveent/RHNM. Last cited on 2009 Sep 25].

7. Corso G, Roncalli F, Marrelli D, Carneiro F, Roviello F. History, pathogenesis, and management of familial gastric cancer: Original study of John XXIII's family. Biomed Res Int. 2013;2013:385132.

8. Apicella M, Corso S, Giordano S. Targeted therapies for gastric cancer: Failures and hopes from clinical trials. Oncotarget. 2017;8:57654-69.

9. Wagner AD, Grothe W, Haerting J, Kleber G, Grothey A, Fleig WE. Chemotherapy in advanced gastric cancer: A systematic review and meta-analysis based on aggregate data. J Clin Oncol. 2006;24:2903-9.

10. Mizrak Kaya D, Harada K, Shimodaira Y, Amlashi FG, Lin Q, Ajani JA. Advanced gastric adenocarcinoma: Optimizing therapy options. Expert Rev Clin Pharmacol. 2017;10:263-71.

11. Van Cutsem E, Moiseyenko VM, Tjulandin S, et al. Phase III study of docetaxel and cisplatin plus fluorouracil compared with cisplatin and fluorouracil as first-line therapy for advanced gastric cancer: A report of the V325 Study Group. J Clin Oncol. 2006;24:4991-7.

12. Kim HS, Kim HJ, Kim SY, et al. Second-line chemotherapy versus supportive cancer treatment in advanced gastric cancer: A meta-analysis. Ann Oncol. 2013;24:2850-4. 
13. Ford HE, Marshall A, Bridgewater JA, et al. Docetaxel versus active symptom control for refractory oesophagogastric adenocarcinoma (COUGAR-02): An open-label, phase 3 randomised controlled trial. Lancet Oncol. 2014;15:78-86.

14. Hironaka S, Ueda S, Yasui H, et al. Randomized, open-label, phase III study comparing irinotecan with paclitaxel in patients with advanced gastric cancer without severe peritoneal metastasis after failure of prior combination chemotherapy using fluoropyrimidine plus platinum: WJOG 4007 trial. J Clin Oncol. 2013;31:4438-44.

15. Thuss-Patience PC, Kretzschmar A, Bichev D, et al. Survival advantage for irinotecan versus best supportive care as second-line chemotherapy in gastric cancer-a randomised phase III study of the arbeitsgemeinschaft internistische onkologie (AIO). Eur J Cancer. 2011;47:2306-14.

16. Cancer Genome Atlas Research Network. Comprehensive molecular characterization of gastric adenocarcinoma. Nature. 2014;513:202-9.

17. Cristescu R, Lee J, Nebozhyn M, et al. Molecular analysis of gastric cancer identifies subtypes associated with distinct clinical outcomes. Nat Med. 2015;21:449-56.

18. Bang YJ, Van Cutsem E, Feyereislova A, et al. Trastuzumab in combination with chemotherapy versus chemotherapy alone for treatment of HER2-positive advanced gastric or gastro-oesophageal junction cancer (ToGA): A phase 3, open-label, randomised controlled trial. Lancet. 2010;376:687-97.

19. Wang X, Chen X, Fang J, Yang C. Overexpression of both VEGF-A and VEGF-C in gastric cancer correlates with prognosis, and silencing of both is effective to inhibit cancer growth. Int J Clin Exp Pathol. 2013;6:586-97.

20. Spratlin JL, Cohen RB, Eadens $M$, et al. Phase I pharmacologic and biologic study of ramucirumab (IMC-1121B), a fully human immunoglobulin G1 monoclonal antibody targeting the vascular endothelial growth factor receptor-2. J Clin Oncol. 2010;28:780-7.

21. Fuchs CS, Tomasek J, Yong CJ, et al. Ramucirumab monotherapy for previously treated advanced gastric or gastro-oesophageal junction adenocarcinoma (REGARD): An international, randomised, multicentre, placebo-controlled, phase 3 trial. Lancet. 2014;383:31-9.
22. Wilke H, Muro K, Van Cutsem E, et al. Ramucirumab plus paclitaxel versus placebo plus paclitaxel in patients with previously treated advanced gastric or gastro-oesophageal junction adenocarcinoma (RAINBOW): A double-blind, randomised phase 3 trial. Lancet Oncol. 2014;15:1224-35.

23. Lee J, Lim T, Uhm JE, et al. Prognostic model to predict survival following first-line chemotherapy in patients with metastatic gastric adenocarcinoma. Ann Oncol. 2007;18:886-91.

24. Catalano V, Graziano F, Santini D, et al. Second-line chemotherapy for patients with advanced gastric cancer: Who may benefit? $\mathrm{Br} \mathrm{J}$ Cancer. 2008;99:1402-7.

25. Wang J, Qu J, Li Z, et al. A prognostic model in metastatic or recurrent gastric cancer patients with good performance status who received first-line chemotherapy. Transl Oncol. 2016;9:256-61.

26. Ock CY, Oh DY, Lee J, et al. Weight loss at the first month of palliative chemotherapy predicts survival outcomes in patients with advanced gastric cancer. Gastric Cancer. 2016;19:597-606.

27. Chen LT, Oh DY, Ryu MH, Yeh KH, Yeo W, Carlesi R. Anti- angiogenic therapy in patients with advanced gastric and gastroesophageal junction cancer: A systematic review. Cancer Res Treat. 2017;3:1-18.

28. Li J, Qin S, Xu J, et al. Randomized, double-blind, placebo-controlled Phase III trial of apatinib in patients with chemotherapy-refractory advanced or metastatic adenocarcinoma of the stomach or gastroesophageal junction. J Clin Oncol. 2016;34:1448-54.

29. Yoon HH, Bendell JC, Braiteh FS, et al. Ramucirumab (RAM) plus FOLFOX as front-line therapy $(\mathrm{Rx})$ for advanced gastric or esophageal adenocarcinoma (GE-AC): Randomized, double-blind, multicenter phase 2 trial. J Clin Oncol. 2014;32 5 Suppl: 4004.

30. Tabernero J, Ohtsu A, Muro K, Van Cutsem E. Exposure-response (E-R) relationship of ramucirumab (RAM) from two global, randomized, double-blind, phase 3 studies of patients (Pts) with advanced second-line gastric cancer. Poster session A (board C19) 2015 gastrointestinal cancers symposium. J Clin Oncol. 2015;33 Suppl 3:121. 